\section{Fractures and survival in multiple myeloma: results from a population-based study}

\author{
Sigrun Thorsteinsdottir, ${ }^{1,2}$ Gauti Gislason, ${ }^{2}$ Thor Aspelund, ${ }^{3}$ \\ Ingigerdur Sverrisdottir, ${ }^{1,2}$ Ola Landgren, ${ }^{4}$ Ingemar Turesson, ${ }^{5}$ \\ Magnus Björkholm ${ }^{6}$ and Sigurður Y. Kristinsson ${ }^{1,2}$
}

${ }^{1}$ Department of Internal Medicine, Landspitali - The National University Hospital of Iceland, Reykjavik, Iceland; 'Faculty of Medicine, University of Iceland, Reykjavik, Iceland; ${ }^{3}$ Centre of Public Health Sciences, Faculty of Medicine, University of Iceland, Reykjavik, Iceland; ${ }^{4}$ Myeloma Service, Division of Hematologic Oncology, Memorial Sloan-Kettering Cancer Center, New York, NY, USA; ${ }^{5}$ Department of Hematology and Coagulation Disorders, Skane University Hospital, Malmo, Sweden and ${ }^{6}$ Department of Medicine, Division of Hematology, Karolinska University Hospital and Karolinska Institutet, Stockholm, Sweden

\section{ABSTRACT}

M ultiple myeloma causes lytic bone lesions and fractures. The impact of fractures on multiple myeloma (MM) survival is unclear. The aim of this study was to evaluate the effect of fractures on survival in MM using data from MM patients diagnosed in Sweden in the years 1990-2013, identified from the Swedish Cancer Registry. Information on date of birth, MM diagnosis, fractures, and death was collected from central registries. A Cox regression model was used to compare survival in patients with and without a fracture at MM diagnosis and another Cox model was used with fracture as a time-dependent variable to assess the effect of fracture on survival after MM diagnosis. Results were adjusted for age, sex, year of diagnosis, and previous fractures. A total of 14,013 patients were diagnosed with $\mathrm{MM}$ during the study, of whom 1,213 (8.7\%) were diagnosed with a fracture at MM diagnosis, and 3,235 (23.1\%) after diagnosis. Patients with a fracture at diagnosis were at a significantly increased risk of death (hazard ratio $=1.28 ; 95 \%$ confidence interval: 1.19-1.37). The risk of death was significantly increased in patients with a fracture after MM diagnosis $(2.00 ; 1.90-2.10)$. The impact of fractures on survival did not change significantly between the two calendar periods 1990-1999 and 2000-2013 (0.98; 0.89-1.08). Our large study shows that MM patients with fractures are at a significantly increased risk of dying compared to those without fractures, which stresses the importance of preventing bone disease in MM.

\section{Introduction}

Multiple myeloma (MM) is a malignant neoplasm of plasma cells in the bone marrow. ${ }^{1,2}$ Skeletal abnormalities are found in the majority of MM patients at the time of diagnosis, and manifestations of bone disease in $\mathrm{MM}$ include osteolytic lesions, osteopenia/osteoporosis, and fractures. ${ }^{3.5}$ Bone disease can be painful and reduces quality of life in $\mathrm{MM}$ patients. ${ }^{6,7}$ In $\mathrm{MM}$ bone disease, the interaction between malignant plasma cells and the bone microenvironment leads to osteoclastic bone destruction, reduced osteoblast function, and blocking of bone repair., This imbalance, along with decreased bone mineral density and treatment-related factors such as treatment with glucocorticoids, can lead to fractures in MM. ${ }^{5,10,11}$ In a population-based retrospective study, MM patients were found to have a 9-fold increase in risk of fractures after $\mathrm{MM}$ diagnosis, as compared to expected fracture rates in the population. ${ }^{5}$ To prevent skeletal-related events, treatment with bisphosphonates is recommended for most patients with $\mathrm{MM}$, and treatment with zoledronic acid has been reported to improve overall survival in MM patients. ${ }^{12,13}$

Previous studies have shown that skeletal-related events (radiation to the bone, a pathologic or osteoporotic fracture, hypercalcemia, spinal cord compression, or surgery to the bone) are associated with reduced survival in both breast cancer and
Ferrata Storti Foundation

Haematologica 2020

Volume 105(4):1067-1073

\section{Correspondence:}

SIGRUN THORSTEINSDOTTIR sth314@hi.is

Received: June 18, 2019.

Accepted: November 28, 2019.

Pre-published: December 2, 2019.

doi:10.3324/haematol.2019.230011

Check the online version for the most updated information on this article, online supplements, and information on authorship \& disclosures: www.haematologica.org/content/105/4/1067

(C)2020 Ferrata Storti Foundation

Material published in Haematologica is covered by copyright. All rights are reserved to the Ferrata Storti Foundation. Use of published material is allowed under the following terms and conditions:

https://creativecommons.org/licenses/by-nc/4.0/legalcode. Copies of published material are allowed for personal or internal use. Sharing published material for non-commercial purposes is subject to the following conditions:

https://creativecommons.org/licenses/by-nc/4.0/legalcode, sect. 3. Reproducing and sharing published material for commercial purposes is not allowed without permission in writing from the publisher. 
prostate cancer. ${ }^{14-16}$ To our knowledge, only three studies have assessed the effect of fractures on survival in MM. In the largest study so far, a total of $513 \mathrm{MM}$ patients diagnosed from 1998 to 2000 and enrolled in a clinical trial were included retrospectively; all patients had DurieSalmon stage III, bone lesions, and a median age of 62 years. No significant difference in survival was observed between MM patients who developed a fracture during follow up as compared to patients that did not develop a fracture. ${ }^{16}$ The second study was a small case-control study in which overall survival was found to be inferior in MM patients with pathologic fractures $(n=24)$ compared to patients with no pathologic fractures $(n=25) .{ }^{17}$ However, in a large study from the UK including patients admitted with plasma cell dyscrasias (MM, plasmacytoma, and plasma cell leukemia), both previous and subsequent fractures were found to be a risk factor for worse survival after first admission for plasma cell dyscrasia. ${ }^{18}$

The aim of our study was to evaluate the impact of fractures and certain subtypes of fractures on survival after $\mathrm{MM}$ diagnosis in a whole population using real-world data on all MM patients diagnosed during a 23-year period in Sweden. We also aimed to compare the effect of fractures on survival in $\mathrm{MM}$ before and after the introduction of novel treatment agents that have greatly improved survival in the MM patient population. ${ }^{19-21}$

\section{Methods}

The Swedish Cancer Registry is a centralized, nationwide registry containing information on patients who have been diagnosed with a malignant disorder in Sweden since January $1^{\text {st }}, 1958$. Every physician and pathologist is obliged by law to report each case of cancer to the registry. The Swedish Cancer Registry contains information on sex, date of birth, date of diagnosis, and histopathologic diagnosis. In a validation study from 2007, the completeness and diagnostic accuracy of the Swedish Cancer Registry was found to be over $93 \%$ for MM patients. ${ }^{22}$ Patients diagnosed with $\mathrm{MM}$ in the period from January 1990 to December 2013 were identified from the Swedish Cancer Registry. Information on date of birth, diagnosis, and death were collected. Information on clinical stage and laboratory results were not available for the patients. Each person in Sweden receives a unique personal identification number that is used to index all major health registers, making it possible to link information in the registries. Information on fractures, using ICD-10 and ICD-9 diagnostic codes (see Online Supplementary Appendix) was obtained from the Swedish Patient Registry, that contains inpatient data from 1987 as well as information on outpatient visits from 2000. Fractures from ten years before $\mathrm{MM}$ diagnosis and then afterwards were included (to adjust for previous fractures before MM diagnosis). Information on date of death was gathered from the Swedish Cause of Death Registry. End of follow up was December 31 ${ }^{\text {st }}, 2013$. The study period was divided into two calendar periods: 1990-1999 and 2000-2013, respectively before and after the introduction of novel treatment options for MM in Sweden that have been shown to improve survival. ${ }^{19,20} \mathrm{~A}$ total of 333 patients were excluded from analysis because of unknown age; 68 of these were patients with a fracture.

A Cox regression model was used to estimate the effect of a fracture at diagnosis (defined as 30 days before or after MM diagnosis) on survival after the time of MM diagnosis. All results were adjusted for age, sex, previous fracture and year of MM diagnosis. Results were presented as hazard ratios (HR) with $95 \%$ confi- dence intervals (CI). $P<0.05$ was considered significant and all statistical analysis was performed in $\mathrm{R}$ version $3.5 .2{ }^{23}$

A landmark analysis was performed by selecting a subset of MM patients alive at six months after MM diagnosis and stratifying patients according to whether they had developed a fracture from the day of MM diagnosis (day 0) until six months after diagnosis or not. Fractures before the day of MM diagnosis were not included in the landmark analysis to avoid any immortal time bias. ${ }^{24} \mathrm{~A}$ Cox regression model was used to assess the association of fracture status at six months and survival, and a Kaplan-Meier graph was generated to visualize the difference in survival between the two groups.

A Cox regression model was used with fracture as a timedependent variable to assess the association of fracture and survival after MM diagnosis (from the day of MM diagnosis). The effect of fracture was assessed for any fracture or a specific subtype of fracture. Specifically, we assessed pathologic (all fractures registered as pathologic fractures), vertebral (both pathologic and others), hip, femoral, humerus, forearm, rib, pelvis, and ankle fractures. Either first fracture or the first subtype of fracture was used in the analysis. The effect of fracture was analyzed for males and females separately for two age groups ( $<70$ years and $\geq 70$ years old) and for the two calendar periods. Results were adjusted for age, sex, time of diagnosis, and previous fractures. To compare the difference in the association of fracture and survival between the two calendar periods, the two age groups, and sexes, the interaction effect of the variable and fracture was assessed in a Cox regression model. Because outpatient visits were included from year 2000, we performed an additional analysis with only inpatient diagnoses of fractures when comparing the calendar periods. Furthermore, we assessed the association of fractures and death after MM diagnosis for two calendar periods after year 2000: 2000-2006 and 2007-2013.

As an additional analysis, four controls, matched by gender, year of birth, and county of residence, were chosen randomly from the Swedish Register of Total Population. All controls were alive and free of $\mathrm{MM}$ at the time of $\mathrm{MM}$ diagnosis for the corresponding patient. Cox regression model was used with fracture as a time-dependent variable from MM diagnosis in the corresponding case to assess the association of fracture and survival in the matched controls. The effect of fracture was assessed for any fracture or a specific subtype of fracture (vertebral, femoral, humerus, rib, or ankle fracture).

\section{Results}

A total of 14,013 patients were diagnosed with $\mathrm{MM}$ in the period from $1^{\text {st }}$ of January 1990 to $31^{\text {st }}$ of December 2013. The median age was 72 years (range $20-99$ years) and $54.9 \%$ were males (Table 1). A total of 4,146 (29.6\%) patients developed a fracture including fractures that occurred a year before MM diagnosis and thereafter, with a sharp rise in fracture diagnoses around the time of $\mathrm{MM}$ diagnosis (Figure 1). Overall, 3,235 (23.1\%) patients were diagnosed with a fracture at the same day or after MM diagnosis (Figure 1). A similar proportion of MM patients developed a fracture in the two calendar periods 19901999 and 2000-2013 (22.2 and 23.7\%, respectively) (Table 1). During the period of follow up, 10,731 (76.6\%) MM patients died, of whom $2,520(77.9 \%)$ were patients with fractures. Median follow-up time from diagnosis to death or end of follow up was 2.4 years (range 1 day-23.8 years). Median overall survival for all MM patients was 3.0 years for the whole study period and improved from 2.6 years 
Table 1. Characteristics of patients with multiple myeloma with and without fractures after multiple myeloma diagnosis.

\begin{tabular}{lccc} 
Variable & All & $\begin{array}{c}\text { Fracture at the same day } \\
\text { or after MM diagnosis }\end{array}$ & $\begin{array}{c}\text { No fractures at the same day } \\
\text { or after MM diagnosis }\end{array}$ \\
$\begin{array}{l}\text { Number of patients } \\
\text { Male/female \% }\end{array}$ & 14,013 & $3,235(23.1 \%)$ & $10,778(76.9 \%)$ \\
\hline Age mean & $54.9 / 45.1$ & $45.7 / 54.3$ & $57.6 / 42.4$ \\
Age median & 70.8 & 70.4 & 70.9 \\
Age range & 72 & 72 & $29-97$ \\
Year at MM diagnosis & $20-99$ & $1,211(22.2 \%)$ & $4,251(77.8 \%)$ \\
$1990-1999$ & 5,462 & $2,024(23.7 \%)$ & $6,527(76.3 \%)$ \\
$2000-2013$ & 8,551 & &
\end{tabular}

MM: multiple myeloma.

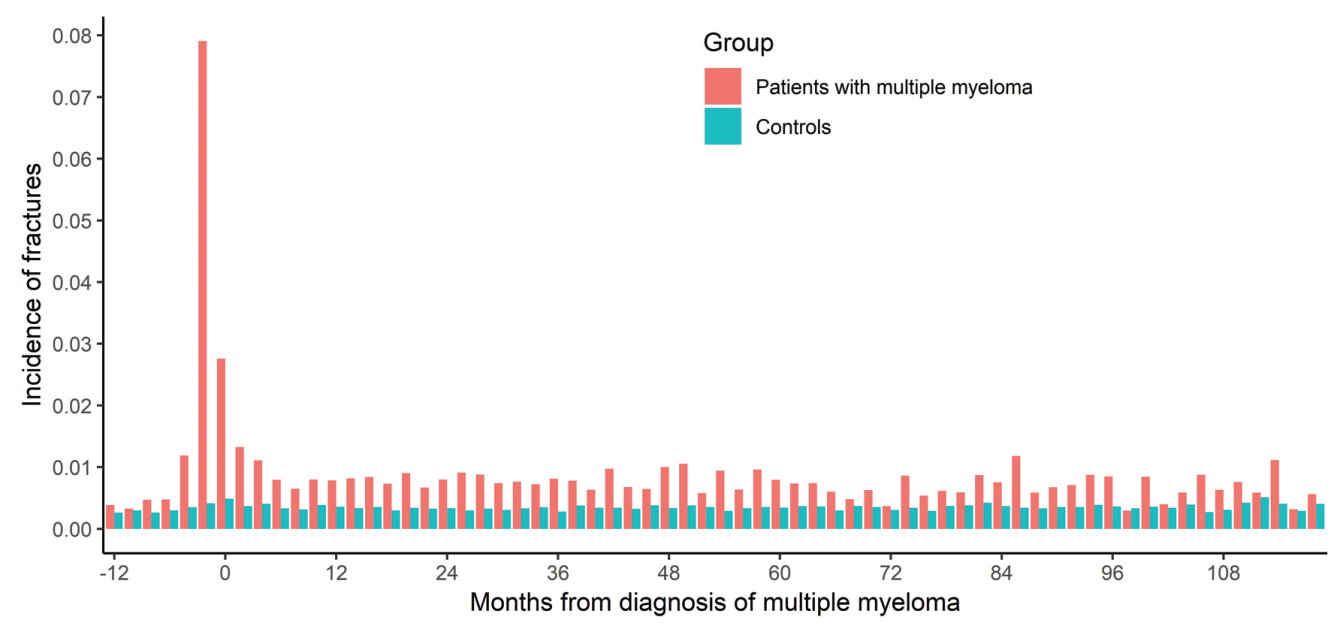

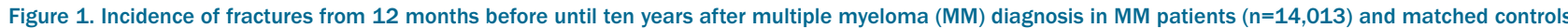
$(n=53,154)$. For each individual, only a single fracture that occurs closest in time from MM diagnosis for the patient or the corresponding case is shown.

in 1990-1999 to 3.3 years in 2000-2013. Median time to first fracture was 1.5 years for $\mathrm{MM}$ patients with a fracture and 10.4 years for all MM patients (Figure 2).

\section{Effect of fractures at diagnosis on survival and landmark analysis}

A total of $1,213(8.7 \%)$ of all MM patients were diagnosed with a fracture at MM diagnosis (within 30 days before or after MM diagnosis). The patients with a fracture at diagnosis were at a significantly increased risk of death compared to those without a fracture $(H R=1.28$; 95\% CI: 1.19-1.37). A total of 11,541 patients were alive six months after $\mathrm{MM}$ diagnosis and were included in the landmark analysis. Of these, $876(7.6 \%)$ patients developed a fracture during the first six months after MM diagnosis (from the day of MM diagnosis). Patients with a fracture during the first six months after MM diagnosis were at a significantly higher risk of death compared to those with no fractures in the first six months $(\mathrm{HR}=1.31$; 95\% CI: 1.20-1.42) (Figure 3).

Effect of fractures on survival after multiple myeloma diagnosis, by subtype, gender, age, and calendar period

The risk of death was significantly increased for patients who developed a fracture after the time of MM diagnosis $(\mathrm{HR}=2.00$; 95\% CI: 1.90-2.10) for all fractures combined. The risk of death was significantly increased in patients who developed all subtypes of fractures after $\mathrm{MM}$ diagnosis; pathologic fracture ( $\mathrm{HR}=2.17$; 95\% CI: 2.03-2.32), vertebral fracture (1.74; 95\% CI: 1.61-1.87), hip fracture (1.99; $95 \%$ CI: 1.82-2.18), femoral fracture (2.62; 95\% CI: 2.32 2.98), humerus fracture (2.57; 95\% CI: 2.31-2.85), forearm fracture $(1.24 ; 95 \% \mathrm{CI}: 1.05-1.46)$, rib fracture $(1.52$; 95\% CI: 1.31-1.77), pelvis fracture (1.99; 95\%CI: $1.74-$ $2.29)$, except ankle fracture (1.07; 95\% CI: 0.79-1.44) (Figure 4). The risk of death was similar for males and females with a fracture as compared to males/females without a fracture $(\mathrm{HR}=2.01 ; 95 \% \mathrm{CI}$ : 1.88-2.16 and 1.99; $95 \% \mathrm{CI}: 1.86-2.13$, respectively). The risk of death for MM patients over 70 years old at diagnosis with a fracture compared to those without a fracture was significantly increased ( $\mathrm{HR}=1.88 ; 95 \% \mathrm{CI}$ : 1.77-2.00). However, in patients under 70 years old at $\mathrm{MM}$ diagnosis, the risk of death in patients with a fracture compared with those without a fracture was more pronounced ( $\mathrm{HR}=2.28$; 95\% CI: 2.11-2.47). The interaction effect of fracture and age group was significant, signifying that the HR for MM patients under 70 years old at diagnosis was significantly higher than for patients $\geq 70$ years old at diagnosis (HR=1.22; 95\% CI: 1.11-1.34; $P<0.01)$.

The risk of death for $\mathrm{MM}$ patients with a fracture after $\mathrm{MM}$ diagnosis was lower in patients diagnosed during 2000-2013 (HR=1.79; 95\%CI: 1.67-1.91) compared to 1990-1999 (2.26; 95\% CI: 2.10-2.42). This difference was 
significant as the interaction effect of a fracture and calendar period was significant for 2000-2013 compared to 1990-1999 (HR=0.85; 95\%CI: 0.77-0.93; P<0.01). When outpatient diagnoses of fractures were excluded, HR for 2000-2013 was 2.08 (95\% CI: 1.94-2.23), HR for 1990-1999 was 2.29 (95\% CI: 2.13-2.46), and the difference between the calendar periods was no longer significant (0.99; 95\% CI: $0.90-1.09 ; P=0.91$ ). When the association of fractures and death after MM diagnosis was assessed for the two calendar periods after year 2000, HR for 2000-2006 was 1.72 (95\% CI: 1.59-1.87), and HR for 2007-2013 was 1.97 (95\% CI: $1.77-2.20)$.
Effect of fracture on survival in matched controls

A total of 53,154 matched controls were included in the study, and 9,897 (18.6\%) developed a fracture during the follow up. The median follow up for all controls was 6.8 years. The risk of death was increased for controls who developed a fracture after the time of MM diagnosis in the corresponding $\mathrm{MM}$ case as compared to controls without a fracture $(\mathrm{HR}=2.02$; 95\% CI: 1.95-2.08) for all fractures combined. The risk of death was increased for controls who developed a vertebral ( $\mathrm{HR}=2.01$; 95\% CI: 1.88-2.15), femoral (2.17; 1.92-2.45), humerus (1.66; 95\%CI: $1.54-$ $1.79)$, and rib (1.62; $95 \% \mathrm{CI}: 1.48-1.78)$ fracture.

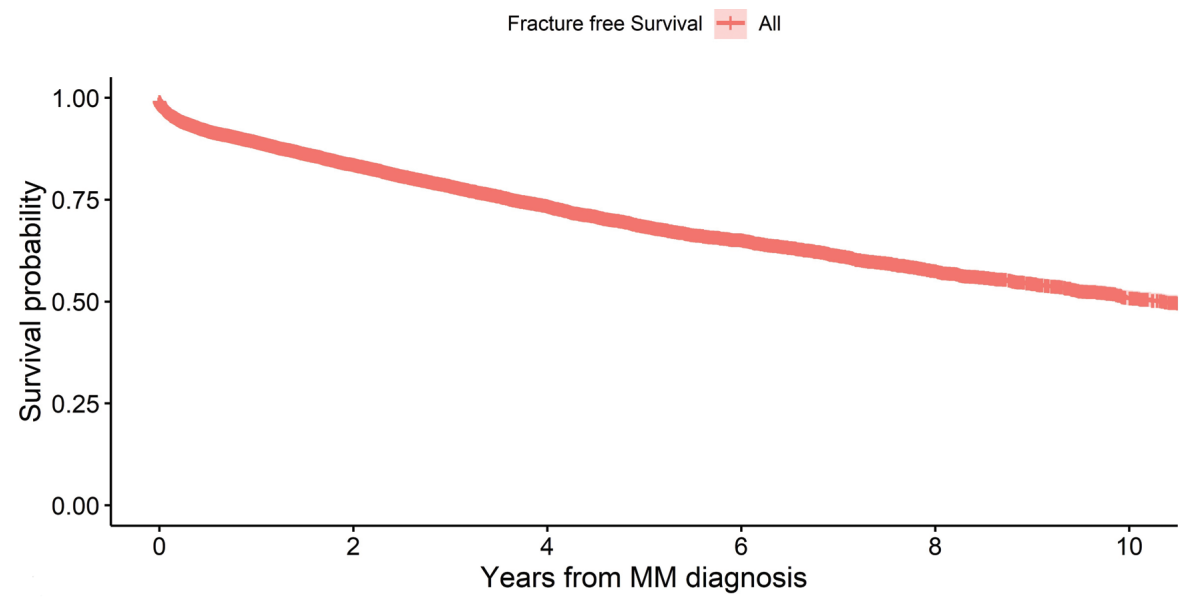

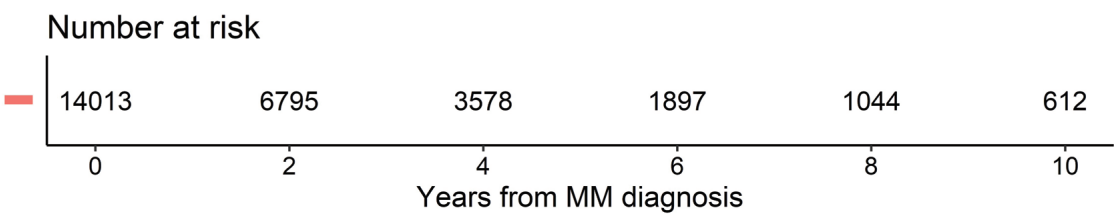

Figure 2. Fracture-free survival in multiple myeloma (MM) patients after MM diagnosis.

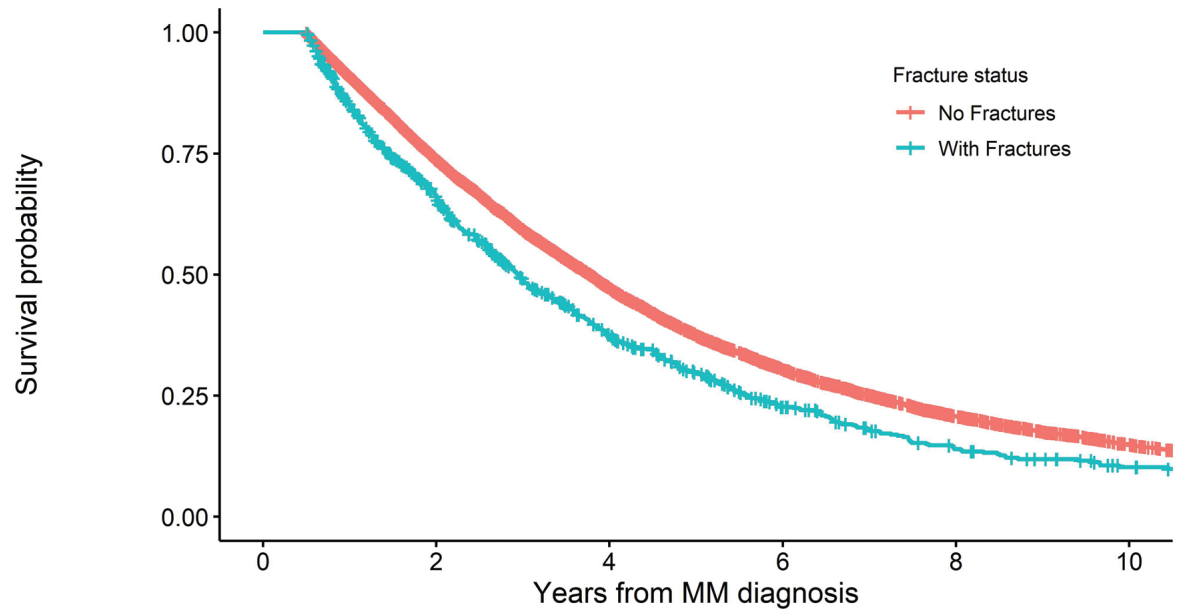

\begin{tabular}{|c|c|c|c|c|c|c|}
\hline$\sum_{\substack{D \\
\pm}}^{\infty}$ & Numbe & & & & & \\
\hline No Fractures & 10665 & 7269 & 4078 & 2259 & 1312 & 787 \\
\hline With Fractures & 876 & 516 & 246 & 109 & 55 & 28 \\
\hline$\pi$ & 0 & 2 & $\begin{array}{c}4 \\
\text { ars fro }\end{array}$ & $\begin{array}{c}6 \\
\text { diano }\end{array}$ & 8 & 10 \\
\hline
\end{tabular}

Figure 3. Landmark analysis showing survival probability in patients with and without a fracture during the first six months after multiple myeloma (MM) diagnosis. 


\section{Discussion}

Our population-based study, including over 14,000 MM patients diagnosed in Sweden from 1990 to 2013, shows that MM patients with a fracture at MM diagnosis are at $28 \%$ higher risk of dying than those without a fracture at diagnosis. Furthermore, after MM diagnosis, patients who develop a fracture are at a 2 -fold increased risk of dying, compared to MM patients who do not develop a fracture. These observations underline the clinical importance of fractures in MM patients in a realworld setting, from diagnosis and throughout the course of the disease.

We found that MM patients with a fracture at diagnosis had an inferior survival compared to MM patients without a fracture at diagnosis which indicates that fractures are a proxy for a more established and/or active disease at diagnosis. We previously showed in a population-based study that individuals with monoclonal gammopathy of undetermined significance (MGUS), a precursor condition preceding $\mathrm{MM}$, had a $74 \%$ increased risk of fractures at five years compared to controls. ${ }^{25}$ Furthermore, our group and others have shown that a prior diagnosis of MGUS or smoldering $\mathrm{MM}$ is associated with improved survival in $\mathrm{MM}$, suggesting that early treatment leads to improved survival. ${ }^{26,27}$ Possibly, a prior diagnosis of a precursor state could reduce the risk of fractures and/or the impact of fractures on survival after MM diagnosis. Our findings underline the importance of thorough evaluation of bone disease in precursor states as well as in active MM.

Our findings of a 2-fold higher risk of dying for MM patients with a fracture compared to patients without fractures demonstrates the significant impact of fractures on patients with $\mathrm{MM}$, and is a higher risk than previously reported in clinical cohorts. ${ }^{16}$ This is, to the best of our knowledge, the first real-world data analysis on the association of fractures and survival in MM. These results were further confirmed in our landmark analysis, where
MM patients with fractures during the first months of the disease had a poorer survival than those without fractures. Our findings are partially in accordance with what has been observed in the general population, where fractures, especially osteoporotic fractures, have been associated with an increased risk of death, although not to the same extent as in MM patients. ${ }^{28-30}$ In patients with metastatic or progressed solid tumors, skeletal-related events have been associated with increased mortality, even when adjusted for stage and/or treatment. ${ }^{14,16}$ Fractures occurring after MM diagnosis may be an indication of aggressive relapse, although we cannot evaluate to what extent fractures are an independent prognostic factor because we do not have clinical data on these patients.

Our analyses on different subgroups of fractures in $\mathrm{MM}$ patients and controls indicate that the impact on survival in $\mathrm{MM}$ reflects both the effect of the fractures themselves as well as the progression of MM. Femoral fractures are well known to increase mortality in the elderly, especially in the first months after the fracture ${ }^{29}$ and were associated with the highest risk of dying in both $\mathrm{MM}$ and the controls in our study. The impact of humerus fractures on survival in MM, on the other hand, seems to reflect the effect of the progression of the disease, since MM patients who developed a humerus fracture after diagnosis had a 2.6fold risk of dying compared to an only 1.7-fold risk in the controls with a humerus fracture. In addition to this, we found that ankle fractures were not associated with survival in $\mathrm{MM}$, highlighting the fact that our findings are specific to MM-related or osteoporotic fractures and not all fractures in general. Our results, therefore, indicate that the effect of fractures on survival in MM is both due to the direct impact of fractures as well as through progression of $\mathrm{MM}$, suggesting that patients with extensive MM bone disease have a more aggressive disease.

We did not find any significant change between the two calendar periods in the risk of death after first fracture in $\mathrm{MM}$. More effective treatment agents were introduced in

All fractures
Males
Females
Age at diagnosis
$<70$ years
>70 years
Calendar period*
$1990-1999$
2000-2013
Fracture subtype
Pathologic
Vertebral***
Hip
Femoral
Humerus
Forearm
Rib
Pelvis
Ankle

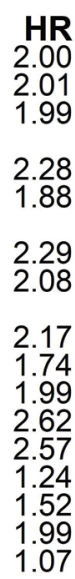

$95 \% \mathrm{Cl}$

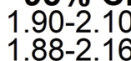

$1.88-2.16$

2.11-2.47

$1.77-2.00$

2.13-2.46

$1.94-2.23$

2.03-2.32

$1.61-1.87$

$1.82-2.18$

2.32-2.98

2.31-2.85

1.05-1.46

1.31-1.77

1.74-2.29

$0.79-1.44$

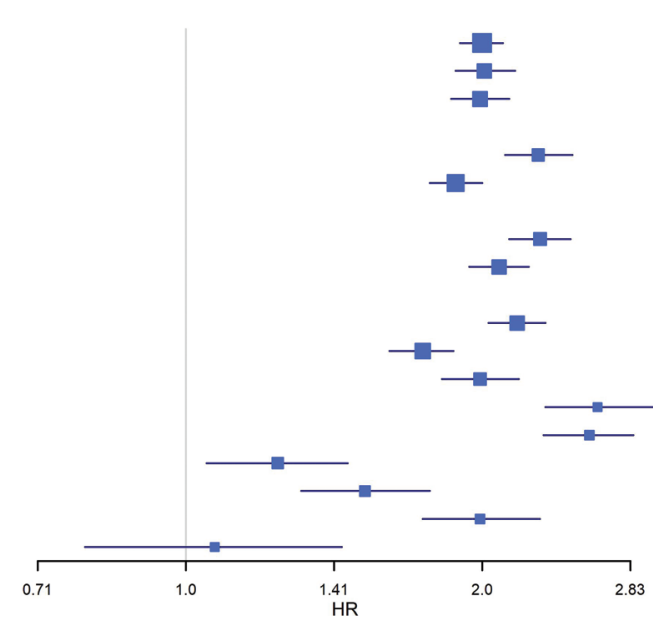

Figure 4. Risk of death in multiple myeloma (MM) patients who developed a fracture after MM diagnosis compared to patients who did not develop a fracture. Fractures as time dependent co-variates, adjusted for age, sex, year of diagnosis and previous fractures. *First inpatient fracture. **All fractures registered as pathologic. $* * *$ All vertebral fractures, both pathologic and others. Cl: confidence interval; HR: hazard ratio; No: number. 
MM after 2000.19-21 These included the immunomodulators thalidomide and lenalidomide, and the proteasome inhibitor bortezomib, that were increasingly used during the later calendar period, and were used in over $80 \%$ of all MM patients in Sweden in 2013. ${ }^{31}$ Previous studies have shown that bortezomib can both inhibit growth of osteoclasts and stimulate osteoblasts, thereby leading to bone healing in $\mathrm{MM}^{32,33}$ Furthermore, the immunomodulators also seem to prompt the bone microenvironment towards bone formation. ${ }^{34,35}$ In addition to this, bisphosphonates, that are a well-established part of the treatment of MM patients, are known to reduce pathologic vertebral fractures, skeletal-related events, and pain. ${ }^{36}$ Moreover, zoledronic acid, that was approved for MM in the later calendar period of the study, has shown a positive effect on overall survival. ${ }^{13}$ In 2010, Swedish national guidelines recommended treatment with bisphosphonates from the time of MM diagnosis for all MM patients, irrespective of the presence of bone disease, and in the years 2008 to 2015, over $70 \%$ of $\mathrm{MM}$ patients received bisphosphonates as part of their disease management. ${ }^{31}$ In our study, even in 2000-2013, when we had information on outpatient visits for fractures for the whole period, we found no change over time in the association of fracture after MM diagnosis and survival. Thus, despite the increasing use of more effective treatment agents and more widespread use of bisphosphonates, fractures are still an important predictor of overall survival.

The strengths of our study include the large populationbased study design, including almost all patients diagnosed with MM in Sweden during a more than 20-year period. ${ }^{22}$ Using these real-world data, all MM patients are included, and the results therefore reflect the actual patient population. Because of the unique identification numbers assigned to every individual in Sweden, we have extensive and accurate follow up for the majority of this large group of patients. Furthermore, the high number of MM patients and fractures yields the study high statistical power to ana- lyze the effect of different subgroups of fractures.

The limitations of our study include the fact that we do not have information on clinical stage, other prognostic factors, or on what treatment the MM patients received. Therefore, it is not possible to determine from our data whether fracture is an independent risk factor for death in MM. Because the fractures occurred after the MM diagnosis, there is a risk of immortal time bias in our survival analysis. To address this, we used both the method of time dependent co-variatesand landmark analysis to accurately analyze the difference in survival between the fractured and unfractured groups. ${ }^{24,37}$ In addition, we do not have access to radiographic images or individual patient records; therefore fractures, especially asymptomatic fractures, might be relatively underreported. In our study, a smaller proportion of patients were diagnosed with a fracture after MM diagnosis than had been previously reported. ${ }^{5}$ The difference between these results might be explained by the study designs, the fact that ours is a large database study using ICDcodes, and that, in the older study, all patient records were searched retrospectively for the occurrence of a fracture. Finally, a limitation of our study is that our follow up ended in 2013; although it would have been interesting to perform our analyses on more recent data, we did not have access to a more up-dated database at the time of the study.

In conclusion, our population-based study shows that MM patients with a fracture at MM diagnosis have an inferior survival than patients without a fracture at diagnosis. Furthermore, we found that MM patients who develop a fracture after the time of MM diagnosis are at a 2-fold risk of dying compared to patients who do not develop a fracture, and that this risk did not decrease significantly after the introduction of more effective treatment agents in MM. Our results stress the importance of preventing bone disease in $\mathrm{MM}$, not only to prevent the morbidity of fractures, but possibly to influence survival.

\section{References}

1. Swerdlow S, Campo E, Harris $\mathrm{N}$, et al. WHO Classification of Tumours of Haematopoietic and Lymphoid Tissues. Vol 2. Revised 4th Edition ed. Lyon, France. IARC; 2016.

2. Rajkumar SV, Dimopoulos MA, Palumbo $A$, et al. International Myeloma Working Group updated criteria for the diagnosis of multiple myeloma. Lancet Oncol. 2014;15(12):e538-548.

3. Kyle RA, Gertz MA, Witzig TE, et al. Review of 1027 patients with newly diagnosed multiple myeloma. Mayo Clin Proc. 2003;78(1):21-33.

4. Kristinsson SY, Minter AR, Korde N, Tan E, Landgren $\mathrm{O}$. Bone disease in multiple myeloma and precursor disease: novel diagnostic approaches and implications on clinical management. Expert Rev Mol Diagn. 2011;11(6):593-603.

5. Melton LJ, 3rd, Kyle RA, Achenbach SJ, Oberg AL, Rajkumar SV. Fracture risk with multiple myeloma: a population-based study. J Bone Miner Res. 2005;20(3):487493.
6. Kiely F, Cran A, Finnerty D, O'Brien T. Selfreported quality of life and symptom burden in ambulatory patients with multiple myeloma on disease-modifying treatment. Am J Hosp Palliat Care. 2017;34(7):671-676.

7. Ramsenthaler C, Osborne TR, Gao W, et al. The impact of disease-related symptoms and palliative care concerns on health-related quality of life in multiple myeloma: a multi-centre study. BMC Cancer. 2016; 16:427.

8. Terpos E, Ntanasis-Stathopoulos I, Gavriatopoulou M, Dimopoulos MA. Pathogenesis of bone disease in multiple myeloma: from bench to bedside. Blood Cancer J. 2018;8(1):7.

9. Yaccoby S. Osteoblastogenesis and tumor growth in myeloma. Leuk Lymphoma. 2010;51(2):213-220.

10. Abildgaard N, Brixen K, Kristensen JE, Vejlgaard T, Charles P, Nielsen JL. Assessment of bone involvement in patients with multiple myeloma using bone densitometry. Eur J Haematol. 1996;57(5):370-376.

11. Dhodapkar MV, Weinstein R, Tricot G, et al. Biologic and therapeutic determinants of bone mineral density in multiple myeloma. Leuk Lymphoma. 1998;32(1-2):121-127.

12. Terpos E, Morgan G, Dimopoulos MA, et al. International Myeloma Working Group recommendations for the treatment of multiple myeloma-related bone disease. J Clin Oncol. 2013;31(18):2347-2357.

13. Morgan GJ, Davies FE, Gregory WM, et al. First-line treatment with zoledronic acid as compared with clodronic acid in multiple myeloma (MRC Myeloma IX): a randomised controlled trial. Lancet. 2010;376(9757):1989-1999.

14. Yong M, Jensen AO, Jacobsen JB, Norgaard M, Fryzek JP, Sorensen HT. Survival in breast cancer patients with bone metastases and skeletal-related events: a population-based cohort study in Denmark (19992007). Breast Cancer Res Treat. 2011;129(2):495-503.

15. Sathiakumar N, Delzell E, Morrisey MA, et al. Mortality following bone metastasis and skeletal-related events among men with prostate cancer: a population-based analysis of US Medicare beneficiaries, 1999. 2006. Prostate Cancer Prostatic Dis. 2011;14(2):177-183. 
16. Saad F, Lipton A, Cook R, Chen YM, Smith M, Coleman R. Pathologic fractures correlate with reduced survival in patients with malignant bone disease. Cancer. 2007;110 (8):1860-1867.

17. Sonmez M, Akagun T, Topbas M, et al. Effect of pathologic fractures on survival in multiple myeloma patients: a case control study. J Exp Clin Cancer Res. 2008;27:11.

18. Mcllroy G, Mytton J, Evison F, et al. Increased fracture risk in plasma cell dyscrasias is associated with poorer overall survival. Br J Haematol. 2017;179(1):61-65.

19. Thorsteinsdottir S, Dickman PW, Landgren $O$, et al. Dramatically improved survival in multiple myeloma patients in the recent decade: results from a Swedish populationbased study. Haematologica. 2018;103(9): e412-e415.

20. Kumar SK, Dispenzieri A, Lacy MO, et al. Continued improvement in survival in multiple myeloma: changes in early mortality and outcomes in older patients. Leukemia. 2014:28(5):1122-1128.

21. Turesson I, Bjorkholm M, Blimark $\mathrm{CH}$, Kristinsson S, Velez R, Landgren O. Rapidly changing myeloma epidemiology in the general population: Increased incidence, older patients, and longer survival. Eur J Haematol. 2018 Apr 20. [Epub ahead of print]

22. Turesson I, Linet MS, Bjorkholm M, et al. Ascertainment and diagnostic accuracy for hematopoietic lymphoproliferative malignancies in Sweden 1964-2003. Int J Cancer. 2007;121(10):2260-2266.

23. R: A language and environment for statistical computing. [computer program]. Vienna, Austria: R Foundation for
Statistical Computing; 2018

24. Gleiss A, Oberbauer R, Heinze G. An unjustified benefit: immortal time bias in the analysis of time-dependent events. Transpl Int. 2018;31(2):125-130.

25. Kristinsson SY, Tang M, Pfeiffer RM, et al. Monoclonal gammopathy of undetermined significance and risk of skeletal fractures: a population-based study. Blood. 2010;116 (15):2651-2655

26. Goyal G, Rajkumar SV, Lacy $\mathrm{MO}$, et al Impact of prior diagnosis of monoclonal gammopathy on outcomes in newly diagnosed multiple myeloma. Leukemia. 2019;33(5):1273-1277.

27. Sigurdardottir EE, Turesson I, Lund SH, et al. The Role of Diagnosis and Clinical Follow-up of Monoclonal Gammopathy of Undetermined Significance on Survival in Multiple Myeloma. JAMA Oncol. 2015;1 (2):168-174.

28. Cooper C, Atkinson EJ, Jacobsen SJ, O'Fallon WM, Melton LJ, 3rd. Populationbased study of survival after osteoporotic fractures. Am J Epidemiol. 1993;137(9): 1001-1005.

29. Katsoulis M, Benetou V, Karapetyan T, et al. Excess mortality after hip fracture in elderly persons from Europe and the USA: the CHANCES project. J Intern Med. 2017;281 (3):300-310.

30. Ray R, Clement ND, Aitken SA, McQueen MM, Court-Brown CM, Ralston SH. High mortality in younger patients with major osteoporotic fractures. Osteoporos Int. 2017;28(3):1047-1052.

31. Blimark CH, Turesson I, Genell A, et al. Outcome and survival of myeloma patients diagnosed 2008-2015. Real-world data on 4904 patients from the Swedish Myeloma Registry. Haematologica. 2018;103(3):506 513.

32. von Metzler I, Krebbel H, Hecht M, et al Bortezomib inhibits human osteoclastogenesis. Leukemia. 2007;21(9):2025-2034

33. Lund T, Soe K, Abildgaard N, et al. First-line treatment with bortezomib rapidly stimulates both osteoblast activity and bone matrix deposition in patients with multiple myeloma, and stimulates osteoblast proliferation and differentiation in vitro. Eur J Haematol. 2010;85(4):290-299.

34. Breitkreutz I, Raab MS, Vallet $S$, et al. Lenalidomide inhibits osteoclastogenesis, survival factors and bone-remodeling markers in multiple myeloma. Leukemia. 2008;22(10):1925-1932.

35. Bolzoni M, Storti P, Bonomini S, et al Immunomodulatory drugs lenalidomide and pomalidomide inhibit multiple myeloma-induced osteoclast formation and the RANKL/OPG ratio in the myeloma microenvironment targeting the expression of adhesion molecules. Exp Hematol. 2013:41(4):387-397.

36. Mhaskar R, Kumar A, Miladinovic B Djulbegovic B. Bisphosphonates in multiple myeloma: an updated network meta-analysis. Cochrane Database Syst Rev. 2017;12:CD003188.

37. Therneau T, Crowson C, Atkinson E. Using time dependent covariates and time dependent coefficients in the cox model 2019; http://cran.r-project.org/web/packages/survival/vignettes/timedep.pdf. Accessed March 29. 